\title{
Long-term habituation is produced by distributed training at long ISIs and not by massed training or short ISIs in Caenorhabditis elegans
}

\author{
CHRISTINE D. O. BECK \\ Baylor College of Medicine, Houston, Texas \\ and \\ CATHARINE H. RANKIN \\ University of British Columbia, Vancouver, British Columbia, Canada
}

\begin{abstract}
To begin an investigation of the cellular processes that underlie long-term memory in the nematode Caenorhabditis elegans, it is first necessary to determine that $C$, elegans is capable of retention over $24 \mathrm{~h}$, and to investigate the factors that may influence the expression of long-term memory. In the present study, the effects of stimuli number, interstimulus interval (ISI), and training procedure on longterm retention of habituation were tested in $C$. elegans. At a long (60-sec) ISI, distributed training sessions produced long-term habituation retained for $24 \mathrm{~h}$, whereas massed training sessions or training with few stimuli did not. When training was performed at a short (10-sec) ISI, long-term habituation was not detectable with testing at either a 10- or a 60-sec ISI. The long-term habituation observed after distributed training sessions at a 60-sec ISI was consistently expressed when the training procedures were varied. Thus it is clear that $C$. elegans can reliably express long-term retention for distributed training sessions at a 60-sec ISI, making the system a candidate for further investigations into the cellular processes supporting memory.
\end{abstract}

The investigation of long-term memory in a simple model system provides an opportunity to define both the behavioral patterns that indicate the operation of memory and the cellular mechanisms that must support memory. The experiments presented here using the nematode Caenorhabditis elegans begin an analysis of the behavioral changes produced by a form of long-term memory, long-term habituation, in this simple organism.

Habituation is a ubiquitous form of learning, found in organisms ranging from protozoa to mammals (Harris, 1943; Thompson \& Spencer, 1966; D. C. Wood, 1988). Habituation may be defined as a decrease in responding that occurs with repeated stimulation (Groves \& Thompson, 1970). It is distinguished from simple fatigue by a number of features, including the expression of dishabituation, which is the facilitation of the habituated (decre-

This work was supported by a Natural Sciences and Engineering Research Council of Canada (NSERC) scholarship to C.D.O.B. as well as operating grants from NSERC and from the Human Frontiers of Science Foundation to C.H.R. We would like to acknowledge Marcus Schatzmann and Jennifer Galloway for their assistance in running a portion of these experiments, Terry Zalynick, Richard Faber, and Marion Buday for scoring data, Catharine Chiba and Stephen Wicks for editorial input, and other members of the lab for useful discussions. Several of the experiments reported here were included in the $\mathrm{PhD}$ dissertation of C.D.O.B, who is affiliated with the Department of Cell Biology at the Baylor College of Medicine. Correspondence should be addressed to C. H. Rankin, Department of Psychology, University of British Columbia, 2136 West Mall, Vancouver, BC, Canada V6T 1Z4 (e-mail: crankin@neuron3.psych.ubc.ca). mented) response by a novel or noxious stimulus, and a sensitivity of both habituation and spontaneous recovery from habituation to interstimulus interval (Groves \& Thompson, 1970; Staddon, 1993). Accumulation of habituation may be observed after an organism has been repeatedly habituated and allowed to spontaneously recover from habituation; this training leads to progressively greater habituation (Groves \& Thompson, 1970; Petrinovich, 1984).

Long-term habituation has been demonstrated in a range of organisms and responses (Bicker \& Hähnlein, 1994; Carew, Pinsker, \& Kandel, 1972; Cerbone \& Sadile, 1994; Leaton \& Supple, 1991). It has been measured in a number of different ways: as a decrease in overall responsiveness (Carew et al., 1972), as an increased rate of habituation (Cheever \& Koshland, 1992), as a decrease in both initial response and overall responsiveness (Leaton \& Supple, 1991), as an increase in the rate of habituation and a decrease in response level (Lozado, Romano, \& Maldonado, 1990), as a decrease in the number of stimuli required for habituation (Bicker \& Hähnlein, 1994), and as a decrease in overall responsiveness (Cerbone \& Sadile, 1994).

The long-term memory for habituation in Aplysia has been studied at both the behavioral and cellular levels (e.g., Bailey \& Chen, 1983, 1988; Carew et al., 1972; Montarolo, Kandel, \& Schacher, 1987). Carew et al. (1972) demonstrated that Aplysia is capable of long-term retention of habituation of two defensive withdrawal responses, the siphon withdrawal response and the gill withdrawal 
response, and demonstrated that the retention of longterm habituation lasts at least 3 weeks. Furthermore, they showed that long-term retention of habituation in the siphon withdrawal response was greater after distributed training sessions than after massed training sessions. Distributed training sessions were given over a series of 4 days, whereas the massed training session was given on a single day. Distributed training sessions contributed to better retention on the 1st test, 1 day later, and after 1 week. In addition, Carew and Kandel (1973) showed that distributed training sessions with blocks of training separated by only $1.5 \mathrm{~h}$ rather than entire days was also effective at producing long-term habituation. The study of long-term memory in Aplysia has led to an examination of the physiological changes in the cells and morphological changes at the synapse (Bailey \& Chen, 1983; Castellucci, Carew, \& Kandel, 1978). Ultrastructural changes at the sensory neuron synapse accompany the induction of long-term memory for habituation (Bailey \& Chen, 1983).

In the present study, the characteristics of long-term habituation in the free-living nematode $C$. elegans will be examined, in order to investigate the processes underlying learning and memory in a new system and search for insights into these processes. $C$. elegans has been widely employed in the study of the structure and function of the genome (W. B. Wood, 1988). The extensive background of information on the organism's biology makes it an excellent candidate for the investigation of cellular and molecular mechanisms of learning and memory (Rankin, Beck, \& Chiba, 1990). The nervous system of $C$. elegans is extremely simple in comparison with other systems studied; all 302 neurons and their cell lineages have been identified, and the connectivity of all the neurons is known (Hall \& Russell, 1991; White, Southgate, Thomson, \& Brenner, 1986). In addition, C. elegans is capable of a variety of forms of behavioral plasticity, ranging from nonassociative to associative learning and from short- to long-term memory (Bargmann, 1993; Gannon \& Rankin, 1995; Rankin et al., 1990).

Habituation of the reversal response in C. elegans exhibits many of the characteristics of habituation described by Groves and Thompson (1970), including dishabituation, spontaneous recovery, and sensitivity to interstimulus interval (ISI). A major finding in our work is that the ISI has important effects on the dynamics of a number of components of habituation, affecting the rate of habituation, the depth of habituation, and the rate of spontaneous recovery from habituation (Broster \& Rankin, 1994; Rankin \& Broster, 1992). Long ISIs (e.g., $60 \mathrm{sec}$ ) lead to slower, shallower response decrement than do short ISIs (e.g., $10 \mathrm{sec}$ ), but also lead to slower recovery from habituation (Rankin \& Broster, 1992). The slower recovery exhibited after training with a long ISI suggests that shortterm retention of habituation is better with longer ISIs (Rankin \& Broster, 1992).

In addition to short-term habituation, Rankin et al. (1990) showed that habituation could be retained and could affect behavior up to $24 \mathrm{~h}$ after the training, a sig- nificant length of time in an organism whose reproductive cycle is 3 days. This finding was used as evidence that C. elegans is capable of a form of long-term memory, long-term habituation. To produce long-term habituation, Rankin et al. used a distributed training sessions procedure in which the experimental group received 100 stimuli in five blocks of 20 stimuli at a 10 -sec ISI separated by periods of rest on Day 1, whereas the control group received only one block of 20 stimuli. A test block of 20 stimuli was given to both groups on Day 2. The Day 2 performance of each group was compared to its Day 1 performance; the experimental group, receiving 100 stimuli, showed a significant decrease from Day 1 to Day 2, whereas the control group, receiving 20 stimuli, did not. This decrease on Day 2 exhibited by the experimental group was considered evidence of long-term habituation. Attempts to replicate these results with a variety of procedures have met with mixed success-in some experiments, long-term habituation has been seen, and in others, it has not (Rankin, unpublished observations). The objectives of the present experiments were to establish a procedure that reliably produces long-term habituation, and to examine the role of several stimulus parameters (stimulus number, stimulus grouping, and ISI) in longterm habituation in C. elegans.

\section{GENERAL METHOD}

\section{Subjects and Materials}

C. elegans Bristol (N2) were maintanned in 4-cm-diameter Petr1 plates filled with $10 \mathrm{ml}$ of Nematode Growth Medium agar. The nematodes fed on E. coli (OP50), as described in Brenner (1974). At 4 days posthatching (the peak of egg laying), subjects were individually placed on labeled agar-filled plates at least $2 \mathrm{~h}$ before training and were maintained on the same plates throughout the procedure. Worms were transferred individually from the breeding plate to the labeled plates with a bent wire pick; simultaneously, small amounts of bacteria sufficient to feed each subject during the study were seeded on each plate.

In each group, 19. 23 worms were run; the data from 10 worms were lost because of technical errors in video recording. The data from a total of 452 worms were included in the analyses of these experiments.

\section{Stimulation and Behavioral Observations}

Observations were made through a stereomicroscope with attached video recording equipment (Wild M3Z, Wild Zeiss Canada; Panasonic Digital 5100 camera; Panasonic AG1960 VCR; NEC monitor). A time-date generator was used to superimpose the experımental time, time of day, and date on the video record (Panasonic WJ-810). The vibrational stimulus used in this work was a 6-Hz train of six taps delivered to the side of the Petrı plate holdıng the subject. Each tap exerted approximately 1-2 N of force on the plate. The stimuli were produced by a mechanical tapper with an electromagnetic relay triggered by a Grass $\mathrm{S} 88$ stımulus generator controlled by the experimenter.

The dependent measure used in this work was a measure of the magnitude of the reversal response to tap, in which the worm, either lying still or swimming forward, responds to a vibrational stımulus by initiating tall-first or backward swimming for a distance. The distance traveled during the reversal response can be quantıfied using stop-frame video analysis and computer-driven digitızıng equipment (Macintosh computer, Bit Pad Plus digitızıng tablet; Macmeasure software). 


\section{Training Procedure}

Worms were trained on Day 1 (4 days posthatching) and tested on Day 2 ( 5 days posthatching). The training procedures consisted of (1) distributed training, in which stimuli were given in blocks separated by a 1 -h rest, (2) massed training, in which stimuli were given consecutively without rest periods, (3) block control training, in which one block of stimuli was given, and (4) single-stimulus control training, in which only a single stimulus was given on Day 1.

The block training control group was included to examine the effects of a small number of stimuli during training on long-term habituation. The single-stimulus control group, which received no training on Day 1 , was included to control for the effects of age and handling. On Day 2, 24-28 h after the end of training on Day 1, all subjects received one block of training. The response level of the single-stimulus control group on Day 2 was expected to be at the same level as the response level of the first block of training on Day 1. Long-term habituation was evident when the Day 2 response levels of a trained group were lower than the Day 2 response level of the single-stimulus control group.

In each of Experiments 1-4, distributed training sessions, massed training sessions, and block and single-stimulus control training procedures were employed. In Experiments 1 and 2, short- and longterm habituation produced by training with 60 stimuli (in three blocks of 20 stimuli each in the distributed training session and massed training session procedures) were examined at two different ISIs. In Experiments 3 and 4, the same procedures were run with 40 stimuli (in four blocks of 10 stimuli each) in an effort to confirm and extend the findings on long-term retention of habituation. The failure of the test for long-term habituation at a 10-sec ISI in Experiments 2 and 4 to conclusively produce long-term habituation led us in Experiment 5 to train at a $10-\mathrm{sec}$ ISI and then test at a $60-\mathrm{sec}$ ISI.

\section{Scoring and Statistical Analyses}

Scoring of responses. Reversal responses were quantified by measuring the distance traveled backward in response to the tap stimulus. A pause in forward swimming was scored as a reversal response of zero, as was no response to the tap stimulus. Increases in forward swimming speed immediately following the tap (accelerations), less than $30 \%$ of the responses, were considered missing data, since the magnitude of accelerations cannot be directly compared with the magnitude of reversal responses. A complete analysis of the effects of training procedure, age, degree of habituation, and expression of long-term habituation on the distribution of the missing data points has been presented elsewhere (Beck, 1995). It was found that the expression of long-term habituation was unrelated to the distribution of missing values, and therefore the distribution of missing values will not be discussed further here.

Treatment of data. Differences in performance during training were examined through analyses of the response magnitudes averaged over blocks of either 5 or 20 stimuli. Obtaining these means decreased some of the variability exhibited in these data and decreased the number of missing data points in the repeated measures analyses. The means of responses were calculated for every subject and then used in the analyses. The comparisons of the mean block magnitudes were used in the analyses of habituation, short-term retention of habituation, and long-term retention of habituation.

General analyses. The statistical package, SPSS (Version 4.0.5 Macintosh), was used for these analyses. Planned comparisons were done by hand. Analyses of variance (ANOVAs) were employed to test for differences between the means of groups and different stages of habituation (see below for a description of the application of ANOVAs to the analysis of short- and long-term habituation). Between-subjects, within-subjects, and mixed-design ANOVAs were employed when appropriate. Appropriate tests were employed to examine the homogeneity of variance and covariance of the data (Lindman, 1991). When the assumption of the homogeneity of vari- ance and covariance was violated, the degrees of freedom of the corresponding $F$ test were adjusted downward using the HuynhFeldt epsilon generated by SPSS.

When appropriate, Fisher's post hoc comparisons were used after significant factorial ANOVAs to assess differences between conditions. Planned comparisons focusing on contrasts that had special significance in the context of the study were employed when the overall ANOVA reached significance. When multiple planned comparisons were used in one analysis, the $\alpha$ level (set at .05) was reduced by dividing by the number of comparisons (Lindman, 1991). Appropriate tests were employed to ensure that the data were robust to assumptions of homogeneity of variance and covariance as was needed (Lindman, 1991).

Analysis of short-term habituation. Short-term habituation was examined during training on Day 1 . To control for variability in responding, the dependent measures used were made up of the means of five consecutive responses. To look at short-term habituation, the effects of different types of training on habituation on Day 1 were compared across the trained groups in Experiments 1 and 2 with a mixed-design ANOVA. The overall effect of training was measured by comparing the mean of the first five responses with the mean of the last five responses. A significant decrease from the initial response level to the final response level on Day 1 was considered evidence of short-term habituation.

The nature of the distributed training session protocols used in Experiments 1 and 2 made it appropriate to expand the analysis of short-term habituation of the distributed training session groups. In the distributed training session procedures of Experiments 1 and 2, stimuli were delivered in three blocks of 20 stimuli with 1 -h rest intervals between the blocks. A two-way repeated measures ANOVA with block (3 levels: Blocks 1, 2, and 3) and training (2 levels: the initial mean of five responses, INIT, and the final mean of five responses, HAB, in each block) was employed. Thus, habituation within blocks (a significant main effect of training, in which the mean of the initial five responses was higher than the mean of the final five responses), the short-term retention of habituation between blocks (a significant main effect of block, in which the levels of responding decreased across blocks), and differences in the habituation expressed in each block (a significant training $\times$ block interaction) were examined through this analysis. Because the expression of spontaneous recovery from habituation was important in the context of the work, a planned comparison was employed to contrast the mean of the last five responses of Block 1 with the mean of the first five responses of Block 2, and likewise for Blocks 2 and 3.

Analysis of long-term habituation. Long-term habituation was defined as a significantly lower Day 2 response level in trained groups than in the single-stimulus control group. The Day 1 response levels were calculated with the means of each worm's responses to the first 20 stimuli on Day 1 in Experiments 1, 2, and 5 and to the first 10 stimuli on Day 1 in Experiments 3 and 4. The Day 2 response levels were calculated with the means of each worm's responses to the 20 stimuli given on Day 2 in Experiments 1, 2, and 5 and to the 10 stimuli given on Day 2 in Experiments 3 and 4. In the analysis, the Day 1 response levels of the trained groups were compared with the Day 2 response level of the single-stimulus group with a factorial ANOVA in order to ensure that there were no age or handling effects and that all trained groups started at comparable response levels. Then the Day 2 response levels of the trained groups were compared with the Day 2 response level of the singlestimulus group with a factorial ANOVA in order to test for the expression of long-term habituation. Fisher's post hoc comparisons were used to detect significant differences between the groups. If the factorial ANOVA showed that there were differences in Day 2 response levels, paired one-tailed $t$ tests were then carried out to test for long-term habituation by testing whether Day 2 scores were significantly lower than Day 1 scores. 


\section{EXPERIMENT 1}

\section{Short- and Long-Term Habituation With 60 Stimuli at a Short ISI $(10 \mathrm{sec})$}

To clarify some of the inconsistent findings from a variety of 10 -sec ISI long-term habituation experiments with different handling and training procedures (Rankin, unpublished observations), both short- and long-term habituation after training at a 10 -sec ISI with massed and distributed procedures were examined.

\section{Method}

Training was delivered at a $10-\mathrm{sec}$ ISI. Four groups were run. In the distributed training sessions group $(n=21), 60$ stimuli were given in three blocks of 20 stimuli each separated by 1-h rest periods. In the massed training session group $(n=21), 60$ stımul were given consecutively. In the 20-stimulus training group $(n=21)$, 20 stimuli were given, and in the single-stimulus control $(n=20)$, only a single stimulus was given. All groups recerved 20 stimuli at least $24 \mathrm{~h}$ later on Day 2.

\section{Results}

Short-term habituation: 60 stimuli, 10-sec ISI. Shortterm habituation was examined across groups by comparing the mean of the initial five responses with the mean of the final five responses on Day 1 . Distributed training (Figure 1A), massed training (Figure 1B), and 20-stimulus control training (Figure $1 C$ ) all produced significant short-term habituation on Day 1 [mixed-design group $\times$ training ANOVA: training, $F(1,55)=173.85, p<.01]$. The groups did not differ from each other in this measure of the expression of short-term habituation [group, $F(2,55)$ $=.02$, n.s.; group $\times$ training, $F(2,55)=.41$, n.s.]. However, it is worth noting that the lack of difference observed between the three procedures may have been produced by a floor effect, since for all groups the mean block magnitude was close to zero by the end of the training runs (mean block magnitude [millimeters] $\pm S E$ to the last five stimuli in distributed training sessions, $0.20 \pm .07$; massed training session, $.09 \pm .03 ; 20$-stimulus control, $0.20 \pm .05$ ).

To examine habituation during distributed training sessions on Day 1, the means of the initial five responses and final five responses of each block were compared in a two-way repeated measures block $\times$ training ANOVA. There was significant habituation within blocks overall [training, $F(1,12)=9.39, p=.01$ ], and this habituation did not vary significantly between blocks [training $X$ block, $F(2,24)=.64$, n.s.]. Interestingly, there was evidence for short-term retention of habituation; the level of responding within blocks exhibited a significant decrease during training on Day 1 [block, $F(1.46,17.5)=5.16, p=$ .02 ; degrees of freedom adjusted with the Huynh-Feldt epsilon]. Because the Mauchly test for sphericity indicated that the assumption of the homogeneity of variances and covariances had been violated, planned comparisons to examine recovery from habituation between blocks of training were not carried out. However, inspection of Fig- ure $1 \mathrm{~A}$ indicates that there appeared to be recovery from habituation between Blocks 1 and 2 and Blocks 2 and 3 .

Long-term habituation: 60 stimuli, 10-sec ISI. No differences between the trained and control groups were evident on Day 1 or Day 2 [see Figure 2: Day $1, F(3,79)=$ 2.39 , n.s.; Day $2, F(3,79)=2.55$, n.s.]. Although the lack of a significant difference between groups was expected on Day 1, the failure to see differences between the groups on Day 2 was not, because long-term habituation should have been apparent as a difference between the Day 2 level of responding among groups. As can be seen in Figure 2, age and handling effects may have depressed response levels on Day 2 in all groups. This was unexpected; the control group that received only a single stimulus on Day 1 should have had Day 2 response levels similar to those of the experimental groups on Day 1. This depression on Day 2 suggests that some factor other than the Day 1 experience was responsible for the depressed responding on Day 2. Such effects would explain the failure to find a significant difference between the trained and untrained Day 2 levels of responding. It is also possible that this short ISI may have generated behavioral levels so low that detection of differences due to training may be difficult. Therefore, at a $10-\mathrm{sec}$ ISI, it was not possible to determine whether training with 60 stimuli produced long-term habituation, although short-term habituation in all trained groups and retention of habituation between blocks of training on Day 1 in the distributed training sessions group were evident.

\section{EXPERIMENT 2}

\section{Short- and Long-Term Habituation With 60 Stimuli at a Long ISI (60-sec)}

As discussed earlier, Rankin and Broster (1992) showed that habituation at a long ISI $(60 \mathrm{sec})$ resulted in slower spontaneous recovery from habituation than did training at a short ISI $(10 \mathrm{sec})$. This is consistent with the findings in a number of other organisms (e.g., rat, Davis, 1970; frog spinal cord, Farel, Glanzman, \& Thompson, 1973; goldfish, Laming \& McKinney, 1990). This observation led to the hypothesis that training at a longer ISI such as $60 \mathrm{sec}$ might also produce better long-term retention of the training than would habituation at a $10-\mathrm{sec}$ ISI. Despite the large body of research on the effects of ISI on short-term habituation, there has been little research on the effect of ISI on long-term habituation. Davis (1970) tested retention of startle response habituation in rats $24 \mathrm{~h}$ following training at either a 2 -sec or a 16-sec ISI and found that the 16-sec group had a lower percentage of startle responses (better retention of habituation) than did the 2-sec trained group. From this data, Davis suggested that, in rats, long ISIs might be superior to shorter ISIs in producing long-term habituation, but that further investigation was necessary. In the present experiment, this hypothesis was tested in $C$. elegans by examining shortand long-term habituation at a 60 -sec ISI. 
A

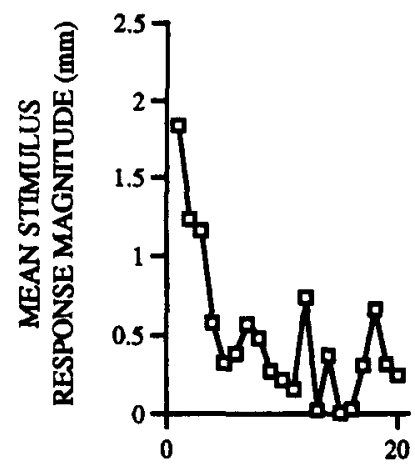

DAY 1

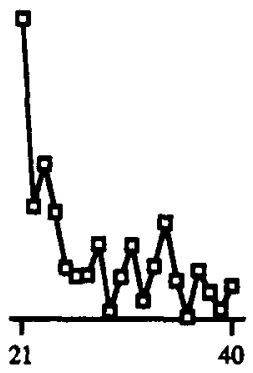

STMULI
DAY 2

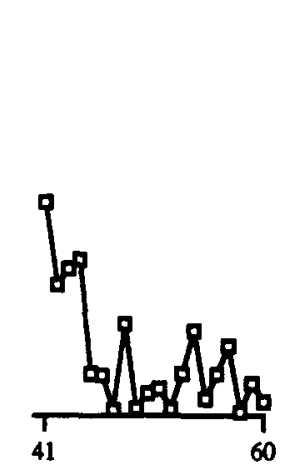

60

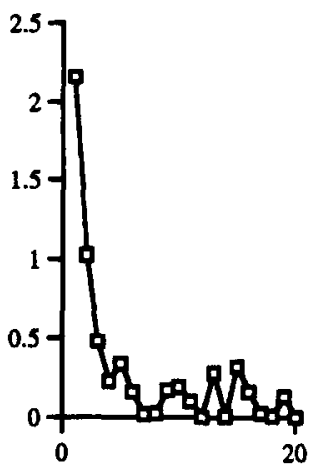

B

DAY 1
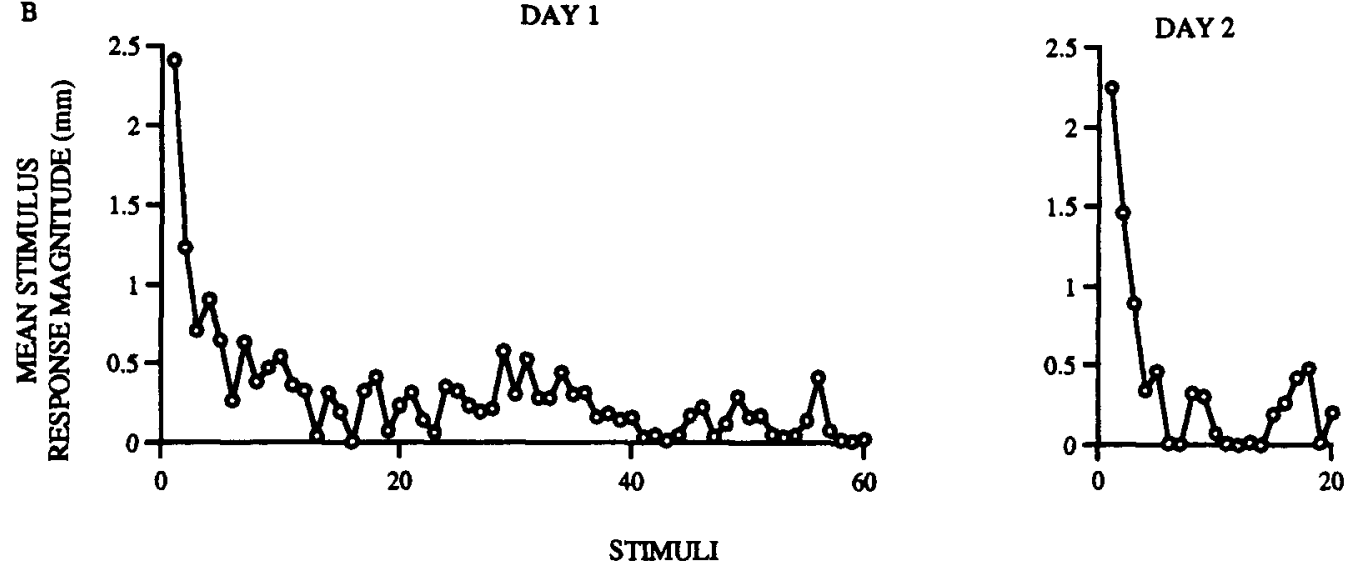

C DAY 1

DAY 2

D SINGLE STIM CONTROL DAY 2
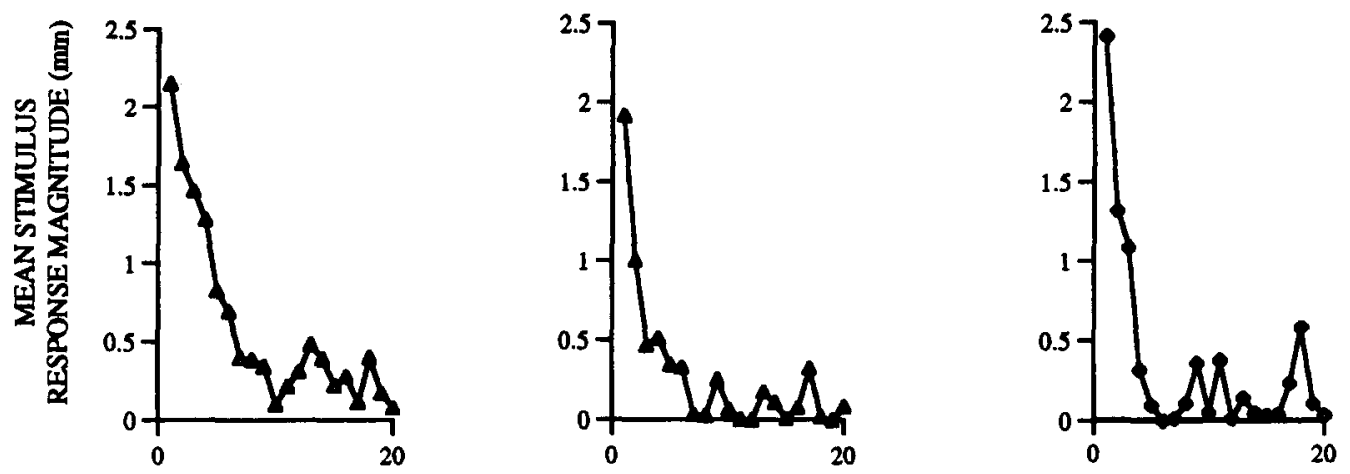

STIMULI

Figure 1. Habituation curves at a 10-sec ISI with 60 stimuli. The mean response magnitude (in millimeters) to each stimulus given during training on Day 1 and testing on Day 2 is shown for subjects receiving distributed training sessions (Figure 1A; $n=21 ; 60$ stimuli in three blocks of 20 stimuli with 1-h rests between blocks), massed training session (Figure 1B; $n=21 ; 60$ stimuli consecutively), 20-stimulus control training (Figure $1 \mathrm{C} ; n=21$; 20 stimuli consecutively), and the single-stimulus control group Day 2 responses (Figure 1D; $n=20$, single stimulus only on Day 1 ). 


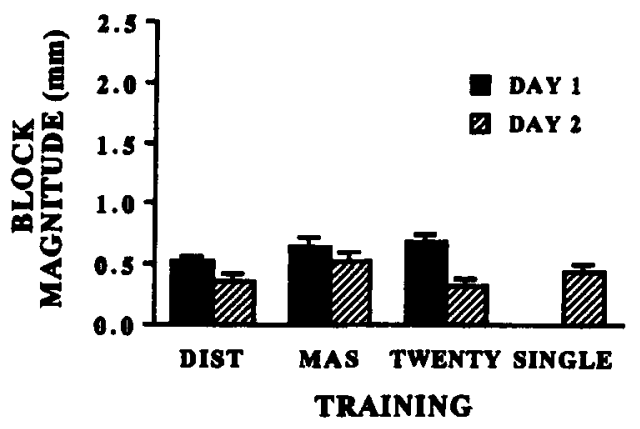

Figure 2. Habituation with 60 stimuli by block at a 10-sec ISI. The figure shows the mean block magnitude (in millimeters) for Day 1 and Day 2 of each of the three groups that received training on Day 1: the distributed training sessions group $(n=21)$, massed training session group $(n=21)$, and 20-stimulus control group $(n=21)$. Also shown is the Day 2 mean block magnitude of the single-stimulus control group $(n=20 ;+S E)$. There were no differences in Day 2 response magnitudes, regardless of training procedure on Day 1.

\section{Method}

Training was delivered at a 60 -sec ISI. The four groups described in Experiment 1 were employed in Experiment 2 as well. In the distributed training sessions group $(n=21), 60$ stimuli were given in three blocks of 20 stimuli each separated by 1 -h rest periods. In the massed training session group $(n=20), 60$ stimuli were given consecutively. In the 20 -stimulus tranning group $(n=20), 20$ stimuli were given, and in the single-stimulus control $(n=19)$, only a single stimulus was given. All groups received 20 stimuli at least $24 \mathrm{~h}$ later on Day 2.

\section{Results}

Short-term habituation: 60 stimuli, 60-sec ISI. At the 60-sec ISI, short-term habituation again resulted with all three training procedures. Comparisons of the response magnitudes of the first and last stimuli on Day 1 in the trained groups show that distributed (Figure 3A), massed (Figure 3B), and 20-stimulus control (Figure $3 \mathrm{C}$ ) training produced a significant decrease in mean response magnitude during training on Day 1 [mixed-design group $\times$ training ANOVA; $\alpha=.025$; training, $F(1,56)=81.64, p<.01]$. Although training procedure did not affect the overall level of responding [group, $F(2,56)=1.96$, n.s.], the groups did differ in the way that they exhibited habituation [group $\times$ training, $F(2,56)=$ $5.46, p<.01]$. In Figure 3, it can be seen that habituation with fewer stimuli (the 20-stimulus control group, Figure $3 \mathrm{C}$ ) led to less profound habituation than did massed or distributed training sessions with 60 stimuli (mean \pm $S E$ in millimeters; distributed training sessions $\mathrm{HAB}$, $0.68 \pm .11$; massed training session HAB, $0.599 \pm .09$; 20 -stimulus control HAB, $1.25 \pm .01$ ).

The habituation during distributed training sessions was considered separately. Overall, training produced habituation during the blocks of training [two repeated measures block $\times$ training ANOVA; training, $F(1,19)=$ $40.79, p<.01]$. There were no significant differences in the way in which habituation was expressed within blocks [block $\times$ training, $F(2,38)=.7$, n.s.]. However, there was evidence of short-term retention of habituation between the blocks [block, $F(2,38)=9.99, p<.01$ ].

Spontaneous recovery between the blocks of training, defined as a significant increase in response magnitude from the end of one block to the beginning of the next, was examined by planned comparisons of Block $1 \mathrm{HAB}$ with Block 2 INIT and Block 2 HAB with Block 3 INIT $(\alpha=.025)$. The recovery between Block 1 and Block 2 was not significant $[F(1,38)=3.39$, n.s. $]$. However, there was evidence of recovery from habituation between Blocks 2 and $3[F(1,38)=14.89, p<.01]$.

Comparisons of short-term habituation on Day 1: 60 stimuli, 10- and 60-sec ISIs. The analyses of shortterm habituation from Experiment 1 and this experiment with training at 10 - and 60 -sec ISIs showed many similarities, though differences were also apparent. At both ISIs, there was habituation on Day 1 in all trained groups; however, at a 60 -sec ISI, the depth of habituation was affected by the type of training, whereas at a 10-sec ISI, it was not. This difference between ISIs is likely to be related to the rapid and profound habituation exhibited during training at a 10 -sec ISI.

As noted in Rankin and Broster (1992), the depth of habituation after training is greater at a short ISI than after a long ISI. In the present experiments, it is clear from a comparison of Figures 1 (short-term habituation at a 10 -sec ISI) and 3 (short-term habituation at a $60-\mathrm{sec}$ ISI) that training at a short ISI produced greater response decrement than did training at a long ISI with the same number of stimuli and the same procedures. The observation that at both the $10-\mathrm{sec}$ and 60 -sec ISIs there was evidence of retention between blocks in the distributed training sessions condition indicates that worms trained at a $10-\mathrm{sec}$ ISI are not insensitive to the effects of this training; however, the effects of this training are not sufficient to produce long-term habituation.

Long-term habituation: 60 stimuli, 60-sec ISI. As can be seen in Figure 4, the single-stimulus control group had a level of responding on Day 2 comparable to the levels of responding on Day 1 of the trained subjects $[F(3,76)=1.46$, n.s. $]$. In addition, when the levels of responding on Day 2 were compared, the training received on Day 1 significantly affected responding $[F(3,76)=$ $3.45, p=.02]$. Fisher's post hoc comparisons indicated that the Day 2 response levels of the distributed training sessions and massed training session groups were significantly lower than that of the single-stimulus control group, whereas the Day 2 response level of the 20-stimulus group was not (Figure 4). However, paired $t$ tests for Day 1 versus Day 2 for distributed training, massed training, and 20-stimulus training showed that only the distributed training sessions group had Day 2 scores significantly lower than Day 1 scores [distributed training, $t(20)=2.42, p=$ .012 ; massed training, $t(19)=1.28$, n.s.; 20 -stimulus 
A
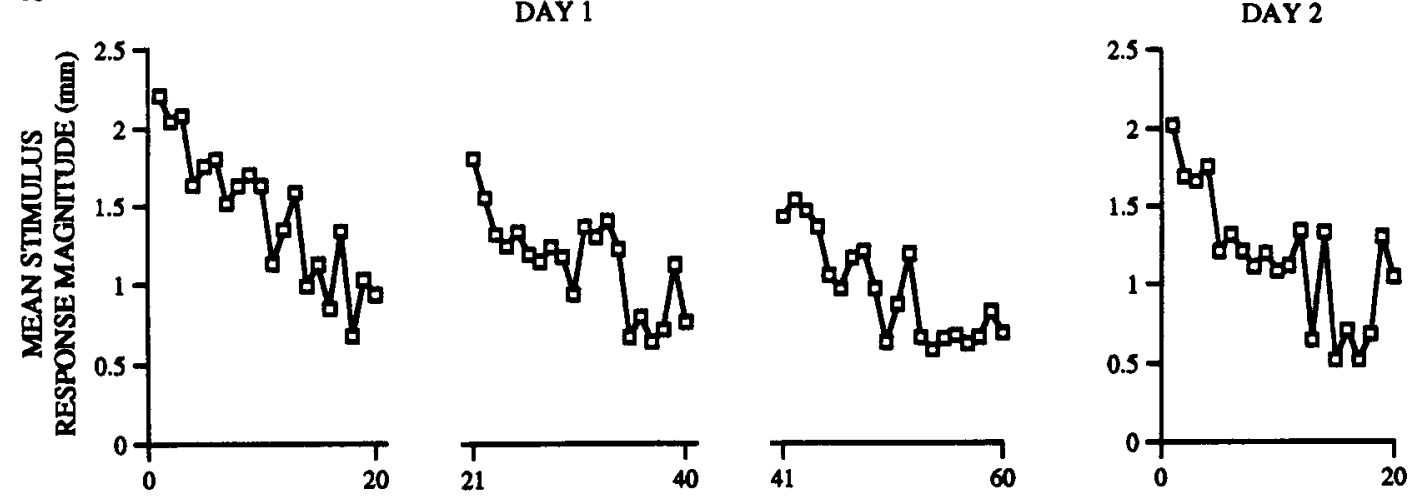

STMMUI

B

DAY 1
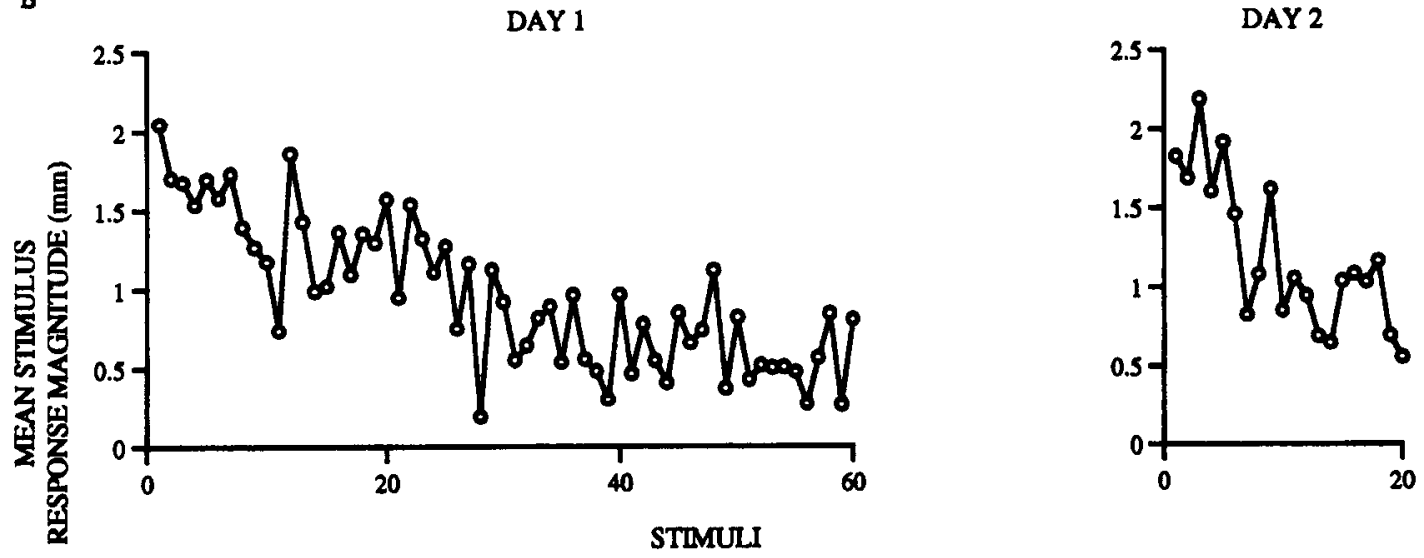

C

DAY 1

DAY 2

D SINGLE STIM CONTROL DAY 2
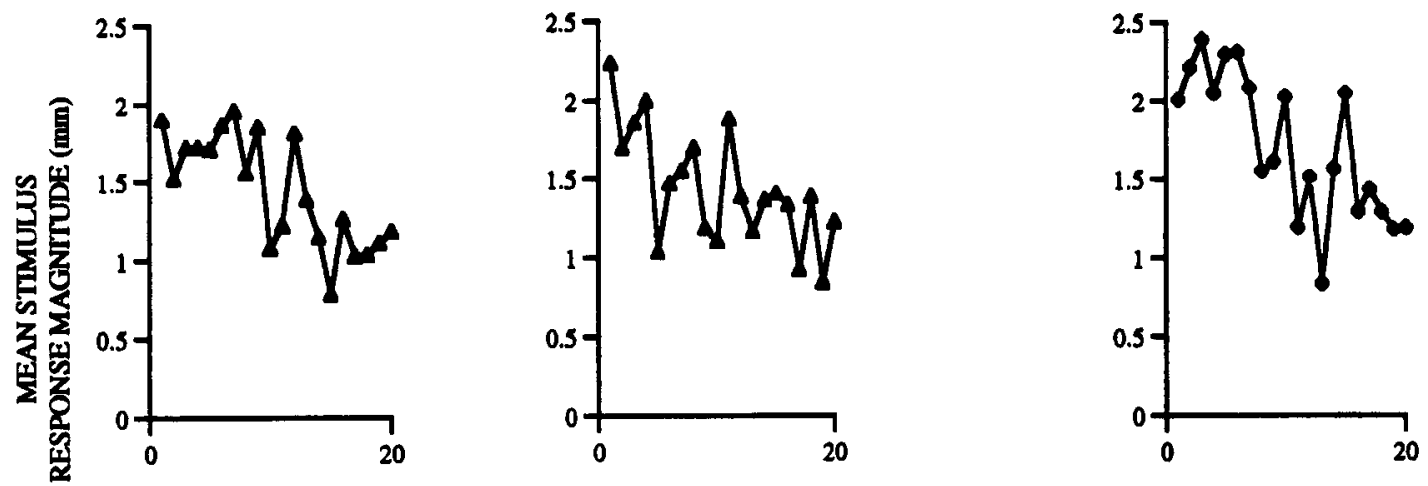

STIMULI

Figure 3. Habituation curves at a 60 -sec ISI with 60 stimuli. The mean response magnitude (in millimeters) to each stimulus given during training on Day 1 and testing on Day 2 is shown for subjects receiving distributed training sessions (Figure 3A; $n=21 ; 60$ stimuli in three blocks of 20 stimuli with 1-h rests between blocks), massed training session (Figure 3B; $n=20 ; 60$ stimuli consecutively), and 20-stimulus control training (Figure 3C; $n=20 ; 20$ stimuli consecutively), along with the mean response magnitude on the single-stimulus control on Day 2 (Figure 3D; $n=19$, single stimulus only on Day 1). 


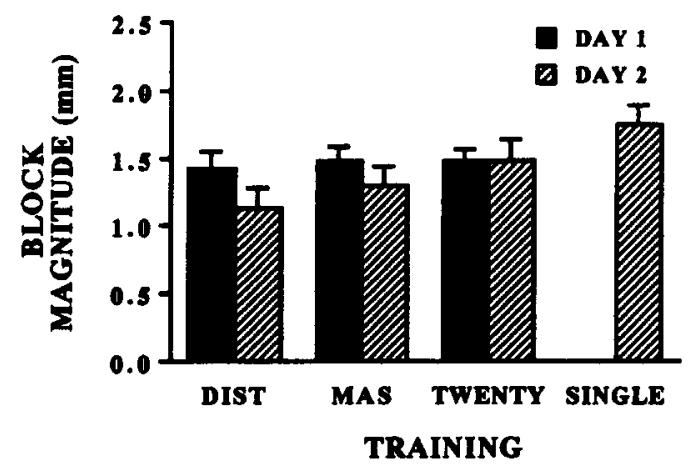

Figure 4. Habituation with 60 stimuli by block at a 60 -sec ISI. The mean block magnitude on Day 1 and Day 2 of the three trained groups, distributed $(n=21 ; 60$ stimuli in three blocks of 20 stimuli with 1-h rests between blocks), massed $(n=20 ; 60$ stimuli consecutively), and 20-stimulus ( $n=20$ ) training, and the mean block magnitude on Day 2 of the single-stimulus control group $(n=19 ; 20$ stimuli consecutively) are shown $(+S E)$. Only the distributed training sessions group showed significant longterm habituation.

training, $t(19)=-.096$, n.s.]. Thus, the distributed training sessions group showed long-term habituation, whereas the massed training session group and the 20-stimulus control group did not.

Comparisons of long-term habituation: 10- and 60-sec ISIs. In Experiment 1, in which training and testing were at a 10-sec ISI, there appeared to be age and handling effects that depressed Day 2 response levels regardless of Day 1 training (Figure 2). These effects were not evident in Experiment 2, in which training and testing were at a 60 -sec ISI (Figure 4). Long-term habituation was expressed after distributed training sessions and testing at a $60-\mathrm{sec}$ ISI; no evidence for long-term habituation was seen in 10-sec ISI tests after any type of training at a $10-\mathrm{sec}$ ISI. It is important to note that these two groups were tested under different conditions (different ISIs), and thus, differences in results might have been due to differences in testing procedures. This possibility will be addressed in Experiment 5. Although this failure to express long-term habituation after training at a 10 -sec ISI cannot be interpreted as a failure to remember training, it is clear that training and testing at a $60-\mathrm{sec}$ ISI is a better procedure to use in the examination of the processes underlying long-term habituation.

In Experiments 1 and 2, distributed training sessions, massed training sessions, and control training procedures were used with 60 stimuli in order to examine long-term habituation. However, the interaction between age and response level when subjects were tested with a 10-sec ISI on Day 2 was unexpected. It was possible that factors specific to procedure, such as the total number of stimuli, the number of training blocks, the duration of training, or the number of stimuli in the test block, contributed these results; therefore, in Experiments 3 and 4, the procedures were altered in order to examine whether these procedural factors were important to the relative response levels observed on Day 2 with and without training.

\section{EXPERIMENT 3}

\section{Long-Term Habituation With \\ 40-Stimulus Training at a 10-sec ISI}

In Experiment 1, there appeared to be an ISI-related age and handling effect on the level of responding on Day 2. In Experiment 3, variations of the training procedure at a 10-sec ISI (i.e., different total number of stimuli, different number of training blocks and different number of stimuli per block) were employed to examine whether this effect was consistently expressed.

\section{Method}

Training was delivered at a 10-sec ISI for five groups of worms. In the distributed training sessions group $(n=22), 40$ stimuli were given in four blocks of 10 stimuli each, with a 1-h rest period after each block during training on Day 1 . In the massed training session group ( $n=20$ ), 40 stimuli were given consecutively. In the 10 stimulus training group $(n=20), 10$ stimuli were given on Day 1 . In the single-stimulus control group $(n=21)$, one stimulus was given, and in the zero-stimulus control group $(n=21)$, no stimuli were given on Day 1 . The zero stimulus control group was included as a comparison for the single-stimulus control group, in case the single stimulus given in the control group produced habituation on Day 2. All groups received a block of 10 stimuli at least $24 \mathrm{~h}$ after training on Day 2.

\section{Results}

As can be seen in Figure 5A, the results follow a pattern similar to those seen in Experiment 1 . There was a significant difference between the Day 1 response levels of the trained groups and the Day 2 response levels of the singlestimulus control group and the zero-stimulus control group [factorial ANOVA with Fisher's post hoc comparisons: $F(4,99)=16.559, p<.01$; Day 1 levels for all trained groups were greater than Day 2 levels for both control groups]. There were no differences between the groups in the Day 2 response levels $[F(4,99)=.351$, n.s.]. As can be seen in Figure 5A, the single-stimulus control group and the zero-stimulus control group were at similar levels on Day 2, confirming that the single stimulus given in the control group was not producing long-term habituation. Thus, despite 10-sec ISI procedures with an overall smaller number of stimuli ( $40 \mathrm{vs} .60)$, a smaller number of stimuli in each block (10 vs. 20), a greater number of training blocks (four vs. three), and fewer stimuli in the test block (10 vs. 20), the effect of age and handling on Day 2 response levels was still apparent. If anything, this was more apparent in Experiment 3, perhaps because in this experiment response level was calculated from the first 10 responses and thus was not as depressed as when it was calculated from 20 responses. In any case it is obvious that with training at a 10 -sec ISI, the effect of age and handling on Day 2 response levels was consistent regardless of variations in the training procedure. 

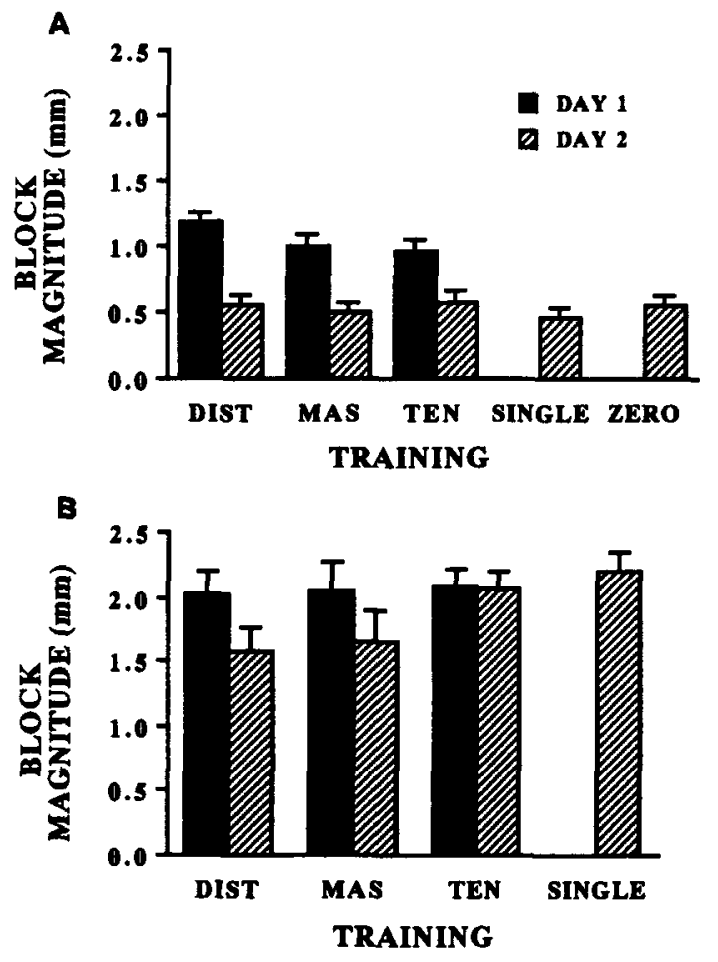

Figure 5. Habituation with 40 stimuli by block at a $10-$ sec and 60-sec ISI. (A) Training at a 10-sec ISI. The mean block magnitude on Day 1 and Day 2 of the three trained groups, distributed ( $n=22 ; 40$ stimuli in four blocks of 10 stimuli with 1-h rests between blocks), massed $(n=20 ; 40$ stimuli consecutively), and 10stimulus $(n=20)$ training and the mean block magnitude on Day 2 of the single stimulus $(n=21)$ and zero-stimulus control groups $(n=21)$ are shown $(+S E)$. Again there were no differences in response magnitude on Day 2 regardless of training procedure on Day 1. (B) Training at a 60-sec ISI. The mean block magnitude on Day 1 and Day 2 of three trained groups, distributed $(n=21)$, massed $(n=20)$, and 10-stimulus $(n=20)$ training and the mean block magnitude on Day 2 of a single-stimulus control group $(n=20)$ are shown $(+S E)$. Only the distributed training sessions group showed significantly smaller responses on Day 2 than on Day 1.

\section{EXPERIMENT 4}

\section{Long-Term Habituation With} 40-Stimulus Training at a 60-sec ISI

In Experiment 2, distributed training sessions with 60 stimuli at a 60 -sec ISI produced significant long-term habituation. In the present experiment, the consistency of these findings is examined with the same variations on the training procedures as in Experiment 3.

\section{Method}

Training was delivered at a 60 -sec ISI for four groups of worms: a distributed training sessions group with 40 stimuli in four blocks of 10 stimuli separated by 1 -h rest periods ( $n=21)$, a massed training session group with 40 stimuli $(n=20)$, a 10 -stimulus control group $(n=20)$, and a single-stimulus control group $(n=20)$. All groups received a test block of 10 stimulı at least $24 \mathrm{~h}$ after training on Day 2.

\section{Results}

As can be seen in Figure 5B, the results in the present experiment were similar to those observed in Experiment 2 . There were no significant differences among the Day 1 response levels of the trained groups and the Day 2 response level of the single-stimulus control group [factorial ANOVA; $F(3,77)=.19$, n.s.]. On Day 2, the response levels of the distributed and massed training session groups were significantly lower than the response level of the single-stimulus control group, but that of the 10-stimulus control group was not [factorial ANOVA; $F(3,77)=2.895, p=.04]$. No other significant differences were evident between groups. Paired one-tailed $t$ tests showed that only the distributed training sessions group had Day 2 response levels significantly below their Day 1 response levels [distributed training sessions, $t(20)=2.82, p=.005$; massed training session, $t(19)=$ 1.31 , n.s.; 10 -stimulus control, $t(19)=.144$, n.s.]. The similarities between these results and those of Experiment 2 suggest that the pattern of results reflects the properties of long-term habituation in C. elegans and not some procedure-specific factor.

\section{EXPERIMENT 5}

\section{Long-Term Habituation With 60-Stimulus Training at a 10-sec ISI and Testing at a 60-sec ISI}

In Experiments 1 and 3 (10-sec ISI), there was a response depression on Day 2 following testing at a $10-\mathrm{sec}$ ISI independent of the type or amount of training on Day 1. It is possible that the training at a 10 -sec ISI was indeed inducing long-term habituation, but that expression was masked by a floor effect reflected in the response depression on Day 2. To eliminate the confound of a test day floor effect obscuring long-term habituation in this experiment, worms that had been trained at a 10-sec ISI were tested at a 60 -sec ISI. Since Experiments 2 and 4 indicated that distributed training sessions produced longterm habituation at a 60 -sec ISI, only distributed training sessions were used in this experiment.

\section{Method}

Five groups were run. In three groups, distributed training sessions with 60 stimuli delivered in three blocks of 20 stimuli each with $1-\mathrm{h}$ rest periods between blocks were given on Day 1 . These three groups consisted of a group that received distributed training sessions on Day 1 at a 10-sec ISI and testing on Day 2 at a 60 -sec ISI ( $n=19 ; 10-60$ group); a group that received distributed training sessions on Day 1 and testing on Day 2 at a 10 -sec ISI $(n=23$; $10-10$ group); and a group that received distributed training sessions on Day $l$ and testing on Day 2 at a 60-sec ISI ( $n=20 ; 60-60$ group). In addition, two control groups were run in which a single stimulus was given on Day 1 and worms were tested on Day 2 with either a 10 -sec or a 60 -sec ISI (single- 10 group, $n=21$; single-60 group, $n=20$ ). For all groups, the test block on Day 2 consisted of 20 stimuli.

\section{Results}

As is evident in Figure 6, distributed training sessions at a 10 -sec ISI did not induce long-term habituation as 


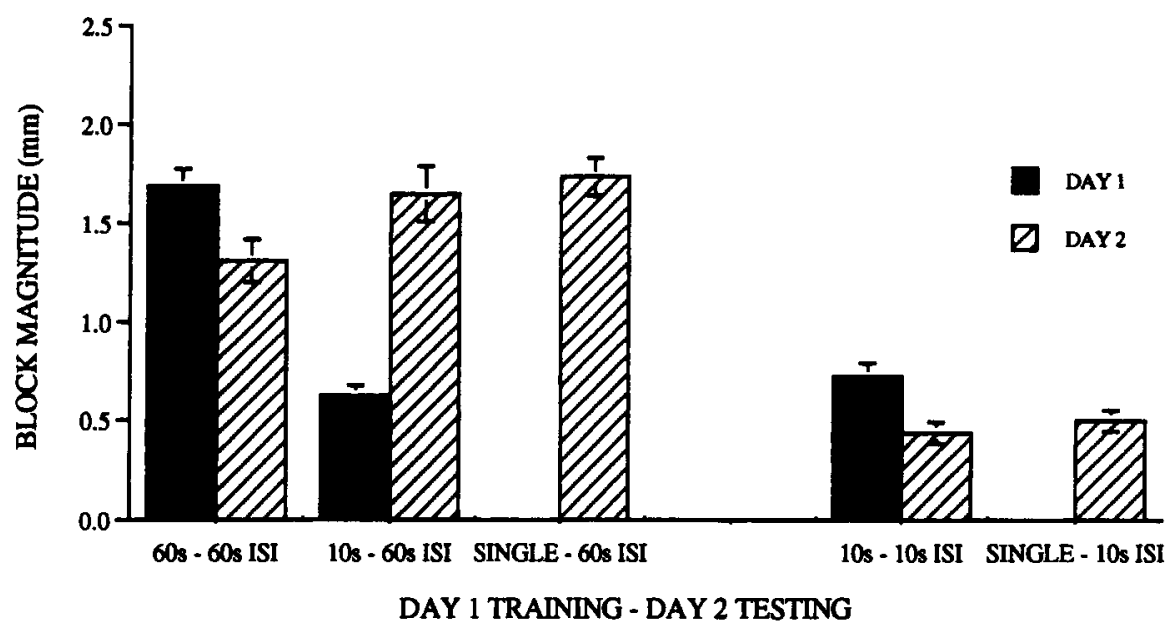

Figure 6. Habituation with distributed training sessions at a 10-sec ISI tested at a 60-sec ISI. The mean block magnitude on Day 1 and Day 2 of the two groups tested at the 60-sec ISI, 60-60 ISI ( $n=20 ; 60$ stimuli at a 60-sec ISI using the distributed training sessions procedure, tested on Day 2 with 20 stimuli at a 60-sec ISI as in Experiment 2), 10-60 ISI ( $n=19$; 60 stimuli at a 10-sec ISI on Day 1 using the distributed training sessions procedure, tested on Day 2 with 20 stimuli at a 60-sec ISI) and the mean block magnitude on Day 2 of the single-stimulus control group tested at the 60-sec ISI, single-60 ISI ( $n=20$; single stimulus on Day 1, tested with 20 stimuli at a 60-sec ISI on Day 2 as in Experiment 2) are shown. In addition, the mean block magnitude on Day 1 and Day 2 of the group trained and tested at the 10-sec ISI 10-10 (n=23;60 stimuli at a 10-sec ISI on Day 1 using the distributed training sessions procedure, tested on Day 2 with 20 stimuli at a 10-sec ISI as in Experiment 1) and the mean block magnitude on Day 2 of the singlestimulus control group tested at the 10-sec ISI, single-10 ISI $(n=21$; single stimulus on Day 1 , tested with 20 stimuli at a 10-sec ISI on Day 2 as in Experiment 1) are shown. Only the group trained and tested at a 60-sec ISI showed significant long-term habituation.

measured by a 60 -sec ISI, whereas long-term habituation after distributed training sessions at a 60 -sec ISI and the Day 2 response depression previously seen with testing at a 10-sec ISI were both replicated. When the Day 1 response levels of groups trained at a 10-sec ISI (10-60 and 10-10 ISI groups) were compared with the Day 2 response levels of the single-10 ISI group, it was evident that there was a response depression with 10 -sec ISI testing on Day 2 without training [factorial ANOVA; $F(2,60)=3.653, p<.05]$. Not surprisingly, there was response depression on Day 2 for all groups tested at a 10 sec ISI: there was no difference between the Day 2 response levels of the trained (10-10 ISI) and untrained (single-10 ISI) groups [unpaired, one-tailed $t$ test; $t(42)=$ $.83, \mathrm{n} . \mathrm{s}$.]. This response depression on Day 2 was not found in the single-stimulus group tested at a 60 -sec ISI (single-60 ISI group); when the Day 2 response level of the single-60 ISI group was compared with the Day 1 response level of the 60-60 ISI group, no difference was observed [unpaired, two-tailed $t$ test; $t(38)=.4$, n.s.]. The Day 2 response level of the 60-60 ISI group was significantly lower than the Day 2 response level of the single-60 ISI group, whereas the Day 2 response level of the 10-60 group was not [factorial ANOVA; $F(2,56)=$ $3.85, p<.05]$. Long-term habituation was demonstrated in this distributed training sessions group (60-60 group) by a paired one-tailed $t$ test showing that Day 2 response levels were significantly below Day 1 response levels $[t(19)=3.78, p=.0007]$. Thus, in Experiment 5, the results from the previous experiments were replicated; more importantly, it was found that distributed training sessions at a 10-sec ISI failed to induce long-term habituation measurable at a 60 -sec ISI. This finding supports the hypothesis that training at a 10 -sec ISI does not induce long-term habituation.

\section{DISCUSSION}

The experiments presented here focus on factors that affect the development of long-term habituation in C. elegans. In Experiments 1, 3, and 5 (training at a 10-sec ISI), an age- and ISI-dependent depression in response levels was observed on Day 2. This Day 2 response level depression, expressed independently of Day 1 training, made it impossible to determine whether training at a 10-sec ISI is capable of producing long-term habituation in C. elegans. In Experiments 2, 4, and 5 (training at a 60-sec ISI), distributed training sessions under several different protocols produced long-term habituation.

In Experiment 5, the question of whether training at a $10-\mathrm{sec}$ ISI could induce long-term habituation was probed further. If habituation at a 10 -sec ISI did in fact induce long-term habituation, it might be detectable when tested at a 60 -sec ISI. The results showed that training did not in fact induce long-term habituation measurable by a 60 -sec ISI test block. This finding strengthens the 
hypothesis that distributed training sessions at a $10-\mathrm{sec}$ ISI do not induce long-term memory for habituation. The failure of training at the short ISI $(10 \mathrm{sec})$ to induce long-term memory for habituation is provocative, considering that short-term memory for habituation, as measured by the rate of spontaneous recovery, is also attenuated at a 10-sec ISI when compared with habituation at a 60-sec ISI (Rankin \& Broster, 1992). In addition, the results support and extend the suggestion by Davis (1970) that long ISIs lead to greater long-term habituation than do short ISIs. These results also support the hypothesis that habituation induced by different ISIs may be mediated by different cellular mechanisms that are recruited differentially by short and long ISIs (Broster \& Rankin, 1994; Rankin \& Broster, 1992).

The finding from Experiments 2 and 4 that distributed training sessions are more effective at producing longterm habituation than a single massed training session is consistent with findings in other organisms. However, it should be noted that, for the $60-\mathrm{sec}$ ISI groups, massed training sessions did produce an effect on Day 2 , since in both Experiments 2 and 4 the Day 2 responses for the massed groups were significantly lower than the Day 2 responses for control groups. It is possible that the memory produced by a massed training session lasts for a shorter period than that produced by distributed training sessions, and that by testing at an earlier time (e.g., $12 \mathrm{~h}$ ), the Day 1-Day 2 response level comparisons would be significant for both groups. In Aplysia, the long-term habituation produced by distributed training sessions lasts much longer than the long-term memory produced by massed training sessions (Carew et al., 1972). Work both with animal systems and with humans suggests that the types of long-term memory resulting from distributed and massed training sessions may be produced by different cellular processes and operate with different behavioral principles (Dellarosa \& Bourne, 1985; Yin et al., 1994). For example, in Drosophila, the effects of protein synthesis inhibition and other interference treatments on memory consolidation for associative learning tasks have been used to differentiate between different forms of memory (Tully, Preat, Boynton, \& Del Vecchio, 1995). Long-term memory (lasting over 7 days) induced by distributed training sessions is disrupted by blocking protein synthesis, whereas long-term memory induced by massed training sessions is not (Yin et al., 1994). In an earlier experiment with $C$. elegans, heat shock was used to disrupt long-term habituation $24 \mathrm{~h}$ following distributed training sessions (Beck \& Rankin, 1995). This effect was hypothesized to be the result of a disruption of protein synthesis during the exposure to heat shock. If, when tested at $12 \mathrm{~h}$ posttraining, the long-term memory for distributed habituation were to be blocked by a treatment such as heat shock or protein synthesis inhibition while the long-term memory for massed training sessions were spared by the same treatment, the contention that distributed and massed training sessions produce qualita- tively different types of memory would be extended to cover memory for nonassociative as well as associative learning.

In summary, the work presented here demonstrates that C. elegans is capable of long-term memory for habituation. With training at a long $60-\mathrm{sec}$ ISI, the expression of long-term habituation can be induced by several different distributed training session procedures. These findings extend the findings of the superiority of distributed training sessions over massed training sessions from a number of organisms for both nonassociative and associative learning to this simple system and provide support for the hypothesis that habituation at short ISIs may be mediated by mechanisms different from habituation at long ISIs. The next step in this work will be to investigate further the differences between massed and distributed training sessions at the $60-\mathrm{sec}$ ISI and to begin a genetic analysis of the memory produced by these different training procedures. Finally, this research indicates that, although $C$. elegans has only 302 neurons, the characteristics of learning and memory that it demonstrates are consistent with those of higher organisms. Therefore C. elegans is a good model system in which to investigate the cellular processes that underlie long-term memory.

\section{REFERENCES}

BaILey, C. H., \& CHEN, M. (1983). Morphological basis of long-term habituation and sensitization in Aplysia. Science, 220, 91-93.

BaIL.EY, C. H., \& CHEN, M. (1988). Long-term sensitization in Aplysia increases the number of presynaptic contacts onto the identified gill motor neuron L7. Proceedings of the National Academy of Sciences, 85, 9356-9359.

BaRGManN, C. I. (1993). Genetic and cellular analysis of behavior in C. elegans. Annual Review of Neuroscience, 16, 47-71.

BECK, C. D. O. (1995). Factors affecting long-term habituation in Caenorhabditis elegans. Unpublished doctoral dissertation, University of British Columbia.

BECK, C. D. O., \& RANKIN, C. H. (1995). Heat-shock disrupts long-term memory consolidation in Caenorhabditis elegans. Learning \& Memory, 2, 161-177.

BICKER, G., \& HÄHNLEIN, I. (1994). Long-term habituation of an appetitive reflex in the honeybee. NeuroReport, 6, 54-56.

BRENNER, S. (1974). The genetics of the nematode Caenorhabditis elegans. Genetics, 77, 71-94.

Broster, B. S., \& RaNkin, C. H. (1994). Effects of changing interstimulus interval during habituation in Caenorhabditis elegans. Behavioral Neuroscience, 108, 1019-1029.

CAREW, T. J., \& KandEL, E. R. (1973). Acquisition and retention of long-term habituation in Aplysia: Correlation of behavioral and cellular processes. Science, 182, 1158-1160.

Carew, T. J., Pinsker, H. M., \& Kandel, E. R. (1972). Long-term habituation of a defensive withdrawal reflex in Aplysia. Science, 175, 451-454.

Castellucci, V., Carew, T. J., \& Kandel, E. R. (1978). Cellular analysis of long-term habituation of the gill-withdrawal reflex of Aplysia californica. Science, 202, 1306-1308.

Cerbone, A., \& Sadile, A. G. (1994). Behavioral habituation of spatial novelty: Interference and noninterference studies. Neuroscience \& Biobehavioral Reviews, 18, 497-518.

Cheever, L., \& Koshland, D. E. (1992). Retention of habituation in PC1 2 cells. Proceedings of the National Academy of Sciences, 89 , 10084-10088

DAvis, M. (1970). Effects of interstimulus interval length and varıabil- 
ity on startle response habituation in the rat. Journal of Comparative \& Physiological Psychology, 72, 177-192.

Dellarosa, D., \& BourNe, L. E., JR. (1985). Surface form and the spacing effect. Memory \& Cognition, 13, 529-537.

Farel, P. B., Glanzman, D. L., \& ThOMPSON, R. F. (1973). Habituation of a monosynaptic response in the vertebrate central nervous system. Journal of Neurophysiology, 36, 1117-1130.

Gannon, T. N., \& Rankin, C. H. (1995). Methods of studying behavioral plasticity in Caenorhabditis elegans. In H. F. Epstein \& D. C Shakes (Eds.), Methods in cell biology: Vol 28. Caenorhabditis elegans: Modern biological analysis of an organism (pp. 205-223). San Diego: Academic Press.

Groves, P. M., \& ThOMPSON, R. F. (1970). Habituation: A dual-process theory. Psychological Review, 77, 419-450.

HALL, D. H., \& RUSSELL, R. L. (1991). The posterior nervous system of the nematode Caenorhabditis elegans: Serial reconstruction of identified neurons and complete pattern of synaptic interactions. Journal of Neuroscience, 11, 1-22.

HARRIS, J. D. (1943). Habituatory response decrement in the intact organism. Psychological Bulletin, 40, 385-422.

LAMING, P. R., \& MCKINNEY, S. J. (1990). Habituation in goldfish (Carassius auratus) is impaired by increased interstimulus interval, interval variability, and telencephalic ablation. Behavioral Neuroscience, 104, 869-875.

Leaton, R. N., \& SupPle, W. F. (1991). Medial cerebellum and longterm habituation of acoustic startle in rats. Behavioral Neuroscience, 105, 804-816.

LINDMAN, H. R. (1991). Analysis of variance in experimental design. New York: Springer-Verlag.

Lozado, M., Romano, A., \& Maldonado, H. (1990). Long-term habituation to a danger stimulus in the crab Chasmagnathus granulatus. Physiology \& Behavior, 47, 35-41.

Montarolo, P. G., Kandel, E. R., \& SchaChER, S. (1987). Long-term heterosynaptic depression of the sensorimotor synapses in culture is produced by the peptide FMRF-amide. Society for Neuroscience Abstracts. 13, 391.

Petrinovich, L. (1984). A theory of habituation and sensitization. In H.V. S. Peeke \& L. Petrinovich (Eds.), Habituation, sensitization and behavior (pp. 17-55). New York: Academic Press.

Rankin, C. H., BeCK, C. D. O., \& ChIBA, C. M. (1990). Caenorhabditis elegans: A new model system for the study of learning and memory. Behavioural \& Brain Research, 37, 89-92.

RANKIN, C. H., \& Broster, B. S. (1992). Factors affecting habituation and recovery from habituation in the nematode Caenorhabditis elegans. Behavioral Neuroscience, 106, 239-242.

Staddon, J. E. R. (1993). On rate-sensitive habituation. Adaptive Behavior, 1, 123-131.

THOMPSON, R. F., \& SPENCER, W. A. (1966). Habituation: A model phenomenon for the study of neuronal substrates of behavior. Psychological Review, 73, 16-43.

Tully, T., Preat, T., Boynton, S. C., \& Del Vecchio, M. (1994). Genetic dissection of consolidated memory in Drosophila. Cell, 79, 35-47.

White, J. G., Southgate, E., Thomson, J. N., \& Brenner, S. (1986). The structure of the nervous system of Caenorhabditis elegans. Philosophical Transactions of the Royal Society of London: Series B, 314, 1-340.

Wood, D. C. (1988). Habituation in Stentor: A response-dependent process. Journal of Neuroscience, 8, 2248-2253.

Wood, W. B. (ED.) (1988). The nematode Caenorhabditis elegans. Cold Spring Harbor, NY: Cold Spring Harbor Laboratory.

Yin, J. C. P., Wallach, J. S., Del Vecchio, E. L., Wilder, E. L., ZHOU, H., QuINN, W. G., \& TulLy, T. (1994). Induction of a dominantnegative CREB transgene specifically blocks long-term memory in Drosophila. Cell, 79, 49-58.

(Manuscript received September 7, 1995; revision accepted for publication April 4, 1997.) 